\title{
Model of a Tunable Hybrid Tamm Mode-Liquid Crystal Device
}

\author{
Maxim V. Pyatnov, Rashid G. Bikbaev, Ivan V. Timofeev, and Stepan Ya. Vetrov \\ Kirensky Institute of Physics, Federal Research Center "Krasnoyarsk Scientific Center", Siberian \\ Branch, Russian Academy of Sciences, Krasnoyarsk, 660036 Russia \\ Siberian Federal University, Krasnoyarsk, 660041 Russia \\ e-mail: MaksPyatnov@yandex.ru
}

\begin{abstract}
A concept of an easily tunable device based on hybrid Tamm modes is proposed. The device can be controlled using a high-sensitivity chiral liquid crystal serving as a mirror. The coupling of the chiral optical Tamm state with the Tamm plasmons is predicted. The Tamm plasmons are excited at different frequencies for the orthogonal linear polarizations, while the chiral Tamm state is excited at only one frequency. The properties of the proposed model are analytically and numerically calculated. The possibility of creating a two- and three-mode laser with tunable characteristics on the basis of the proposed model are discussed.
\end{abstract}

The Tamm modes, including Tamm plasmons (Tamm plasmon polaritons) and optical Tamm states, have been intensively studied for more than a decade [1-3]. The interest in these modes is due to their potential for application in lasers, emitters [4-6], absorbers [7.8], sensors [9], and photovoltaic [10], topological photonic [11, 12], and other devices [13-16].

The Tamm modes can be strongly coupled with each other or with resonances of a different nature, e.g., the cavity modes, exciton polaritons, and surface plasmons [1721]. Such hybrid modes are characterized by the resonance quasi-crossing at the detuning of the position of one of the resonances, which is widely used in sensors [22, 23].

Liquid crystals are promising for rearranging the position of Tamm plasmons [14, 24]. They can be used both as service components for changing the optical properties and as Bragg reflectors. In the latter case, as a rule, cholesteric liquid crystals (CLCs) are used. In contrast to scalar dielectric multilayers, CLCs have the only band gap at the normal incidence, which only exists for the circular polarization of light coinciding with the cholesteric helix twist [25].

In contrast to the case of metals or conventional dielectric materials, the circularly polarized radiation reflected from a CLC preserves its polarization direction. Therefore, it is difficult to observe the Tamm modes localized between a CLC and a metallic layer $[13,26,27]$. A possible way of observing them is to use of a polarization preserving (handedness preserving or chiral) anisotropic mirror (PPAM) [28-30].

Here, we discuss the design of a device based on hybrid Tamm modes. The proposed structure supports the Tamm modes of two types: the Tamm plasmon (TP) and 
the chiral optical Tamm state (COTS). It is proposed to tune the COTS spectral position by changing the parameters that are only inherent in a chiral medium, such as a CLC.

\section{DESIGN OF THE DEVICE}

Figure 1 shows a design of the proposed device. A multilayer anisotropic mirror is bounded by a metallic film on one side and by a cholesteric layer on the other. The mirror is a structure that preserves the polarization of the incident light. It consists of $N$ periods of alternating uniaxial dielectric layers with refractive indices $n^{m}{ }_{\mathrm{e}}$ and $n^{m}{ }_{\mathrm{o}}$. Each layer is rotated relative to the previous one by $90^{\circ}$. The PPAM period thickness is $2 a$. A cholesteric is characterized by extraordinary and ordinary refractive indices $n_{\mathrm{e}}$ and $n_{\mathrm{o}}$ and helix pitch $p$. The angle between the CLC director and the optical axis of the PPAM layer adjacent to it is designated by $\varphi$. The PPAM layer adjacent to the metallic film with thickness $d_{m}$ was assumed to have different thickness $d$. The structure is surrounded by a medium with the refractive index equal to the averaged CLC refractive index $n_{\mathrm{ext}}=\left(n_{\mathrm{e}}+n_{\mathrm{o}}\right) / 2$. Such an approach makes it possible to weaken the effect of Fresnel reflection on the spectral properties of the sample.

The two Tamm modes that can be excited in the proposed structure are the TP localized at the metal-mirror interface and the COTS localized at the PPAM-cholesteric interface. It is expected that the change in the parameters of the mirror will allow us to tune the coupling between the modes.

At the interface between the metallic film and the multilayer Bragg reflector, the role of which in the investigated model is played by the PPAM, a TP can be excited, the frequency of which is determined from the condition [2]

$$
r_{\mathrm{ME}} r_{\mathrm{PPAM}} e^{2 \mathrm{ix}}=1 \text {, }
$$

where $r_{\mathrm{ME}}$ and $r_{\mathrm{PPAM}}$ are the amplitude reflectivities of the metallic and PPAM layers and $\chi=n_{\mathrm{a}} d \omega / c$ is the phase variation in the wave with frequency $\omega$ propagating in the boundary layer with thickness $d$ and refractive index $n_{\mathrm{a}}$.

The amplitude reflectivity of a multilayer consisting of $N$ periods is determined using the equation [31]

$$
r_{\mathrm{PPAM}}=\frac{C U_{N-1}}{A U_{N-1}-U_{N-2}} .
$$

In Eq. (2), $U_{N}=\frac{\sin 2 a(N+1) K}{\sin 2 a K}$, where $K=\frac{1}{2 a} \arccos \left[\frac{1}{2}(A+D)\right]$ is the Bloch wavenumber.

Since we only analyze the case of normal incidence, the elements $A, B, C$, and $D$ of the transfer matrix for one cell, which relate the amplitudes of plane waves in the first 
layer of the unit cell to the amplitudes in the neighboring unit cell can be written as

$$
\begin{aligned}
& A=e^{i k_{1 z} a}\left[\cos k_{2 z} a+\frac{1}{2} i\left(\frac{k_{2 z}}{k_{1 z}}+\frac{k_{1 z}}{k_{2 z}}\right) \sin k_{2 z} a\right], \\
& B=e^{-i k_{1 z} a}\left[\frac{1}{2} i\left(\frac{k_{2 z}}{k_{1 z}}-\frac{k_{1 z}}{k_{2 z}}\right) \sin k_{2 z} a\right], \\
& C=e^{i k_{1 z} a}\left[-\frac{1}{2} i\left(\frac{k_{2 z}}{k_{1 z}}-\frac{k_{1 z}}{k_{2 z}}\right) \sin k_{2 z} a\right], \\
& D=e^{-i k_{1 z} a}\left[\cos k_{2 z} a-\frac{1}{2} i\left(\frac{k_{2 z}}{k_{1 z}}+\frac{k_{1 z}}{k_{2 z}}\right) \sin k_{2 z} a\right],
\end{aligned}
$$

where $k_{1 z}=(\omega / c) n_{e}^{2}, k_{2 z}=(\omega / c) n_{o}^{2}$ are the wavenumbers of the first and second media, respectively and $2 a$ is the PPAM period.

The amplitude reflectivity $r_{\mathrm{ME}}$ of the metallic layer can be determined using the Airy formula [32]:

$$
r_{M E}=\frac{r_{12}+r_{23} e^{2 i k}}{1+r_{12} r_{23} e^{2 i k}} .
$$

In Eq. (4), $r_{12}=\frac{n_{e x t}-n_{m}}{n_{e x t}+n_{m}}, r_{23}=\frac{n_{m}-n_{a}}{n_{m}+n_{a}}, k=\mathrm{d}_{\mathrm{m}} \omega n_{m}$. Here, $n_{\mathrm{a}}$ is the refractive index of the layer adjacent to the metal and $d_{m}$ and $n_{m}$ are the thickness and refractive index of the metal, which is expressed, in the Drude approximation, by the formula

$$
n_{m}^{2}=\varepsilon_{0}-\frac{\omega_{p}{ }^{2}}{\omega(\omega+i \gamma)},
$$

where $\varepsilon_{0}$ is the contribution of the ionic core, $\omega_{\mathrm{p}}$ is the plasma frequency, and $\gamma$ is the reciprocal relaxation time.

Near the Bragg frequency of the mirror, the amplitudes $r_{\mathrm{PPAM}}$ and $r_{\mathrm{ME}}$ are almost unity. We designate the phases of the waves reflected from the PPAM and metal by $\varphi_{\mathrm{PPAM}}$ and $\varphi_{\mathrm{ME}}$, respectively, and write Eq. (1) in the form

$$
0=\varphi_{P P A M}+\varphi_{M E}+2 \chi \text {. }
$$

A feature of the investigated model is that, at the interface between the metal and the PPAM, the TP is excited at different frequencies for the orthogonal linear polarizations of the incident light.

The frequency of the COTS localized at the interface between two media with reflectivities $r_{\mathrm{PPAM}}$ and $r_{\mathrm{CLC}}$ and the angle $\varphi$ between the optical axes can be found similarly from the equation [30]

$$
r_{P P A M} r_{C L C} \mathrm{e}^{2 \mathrm{i} \varphi}=1 \text {, }
$$

where $r_{\mathrm{PPAM}}$ and $r_{\mathrm{CLC}}$ are the PPAM and CLC amplitude reflectivities and $\varphi$ is the angle between the CLC optical axis and the mirror layer adjacent to it.

The amplitude reflectivity of a cholesteric is [25] 


$$
r_{C L C}=\frac{i \delta \sin q d}{\left(q \tau / \kappa^{2}\right) \cos q d+i\left((\tau / 2 \kappa)^{2}+\left(q / \kappa^{2}\right)^{2}-1\right) \sin q d} .
$$

Here, $\delta=\frac{n_{e}^{2}-n_{o}^{2}}{n_{e}^{2}+n_{o}^{2}}, \kappa=\frac{\omega}{c} \sqrt{\frac{n_{e}^{2}+n_{o}^{2}}{2}}$, and $\tau=\frac{4 \pi}{p}$ is the vector of the reciprocal CLC lattice.

The wave vector of the mode diffracting in the CLC, which has a band gap, is

$$
q=\kappa \sqrt{1+\left(\frac{\tau}{2 \kappa}\right)^{2}-\sqrt{\left(\frac{\tau}{\kappa}\right)^{2}+\delta^{2}}} .
$$

Near the Bragg frequency, the amplitudes $r_{\text {PPAM }}$ and $r_{\text {CLC }}$ are almost unity. We designate the phases of the waves reflected from the PPAM and CLC by $\varphi_{\text {PPAM }}$ and $\varphi_{\text {CLC }}$ and write Eq. (7) in the form

$$
0=\varphi_{P P A M}+\varphi_{C L C}+2 \varphi .
$$

Thus, in the proposed structure (Fig. 1), the frequencies of single excited resonances are determined using Eqs. (6) and (10). The two modes are localized at different PPAM boundaries. It is expected that, at small PPAM thicknesses, the electromagnetic fields of the localized modes will overlap and the interaction between the resonances will lead to their splitting, when their frequencies coincide.

We will consider mainly the case of the normal incidence of circularly polarized light onto the structure. This is due to the fact that, in cholesterics, the quasi-circular polarization is their own [25]. In addition, the circular polarization can always be presented as a sum of orthogonal linear polarizations.

\section{RESULTS}

\section{Tamm Plasmons}

First, we discuss the features of the excitation of pure TPs in the proposed model (Fig. 1). For this purpose, we should consider the model without a CLC. The TP position is conventionally tuned by changing thickness $d$ or refractive index $n_{a}$ of the layer adjacent to the metallic film. For light of each of the linear polarizations propagating along the PPAM optical axes, the PPAM is an anisotropic photonic crystal; therefore, at the orthogonal linear polarizations of the incident light, the TPs are excited at different wavelengths. Figure 2 shows the transmittance spectrum for a pure (without cholesteric) TP structure with different thicknesses $d$ of the layer adjacent to the metallic film. The calculation was made using the Berreman matrix method [33]. In the simulation, we used PPAM refractive indices of $n^{m}{ }_{e}=1.71$ and $n^{m}{ }_{o}=1.54$, a period of $2 a=200 \mathrm{~nm}$, and a number of periods of $N=20$. The metallic film thickness was taken to be $d_{\mathrm{m}}=50 \mathrm{~nm}$ and the other film parameters were $\varepsilon_{0}=5, \hbar \omega_{\mathrm{p}}=9 \mathrm{eV}$, and $\hbar \gamma=0.02 \mathrm{eV}$, which are typical of silver.

The white and red lines in Fig. 2 show the TP wavelengths for the TE and TM polarizations of the incident light calculated using Eq. (6). At the parameters used, the 
PPAM band gap center is located at a wavelength of $650 \mathrm{~nm}$. At $d=184 \mathrm{~nm}$, the TP is excited exactly at $650 \mathrm{~nm}$ for the TM polarization of the incident light.

Changing the metallic film thickness, we found that the maximum transmittance at the TP frequency is observed at $d_{\mathrm{m}}=50 \mathrm{~nm}$. At smaller thicknesses, the reflectivity of the film is low and, at larger thicknesses, the absorption is high.

The PPAM period variation leads to the change in the phase of the reflectivity $\varphi_{\text {PPAM }}$, which results in a slight shift of the TP frequency. At $2 a=600 \mathrm{~nm}$, the TP is excited for the TM polarization of the incident light at a wavelength of $648.6 \mathrm{~nm}$ and, at $2 a=1000 \mathrm{~nm}$, at a wavelength of $648.8 \mathrm{~nm}$.

\section{Chiral Optical Tamm State}

If the proposed structure (Fig. 1) does not contain a metallic layer, then, the COTS localized at the PPAM/CLC interface can be excited in it. Figure 3 shows a transmittance spectrum of the PPAM/CLC structure with different CLC helix pitches, a thickness of 4 $\mu$, and refractive indices identical of those of the PPAM, i.e., $n_{e}=1.71$ and $n_{o}=1.54$. The parameters correspond to the mixture of a chiral center (Merck, S-811) and a nematic liquid crystal. The crystal was assumed to be right handed, i.e., the right-hand circular polarization could reflect from it diffracted.

In the spectrum, we can see a transmission peak corresponding to the COTS. The magenta line shows the COTS position according to Eq. (10) at different CLC helix pitches. At a helix pitch of $400 \mathrm{~nm}$, the CLC and PPAM band gaps coincide; the COTS is excited exactly at $650 \mathrm{~nm}$. The COTS frequency significantly depends on the angle $\varphi$ between the optical axes at the interface. At $\varphi=\pi / 4$, the COTS is excited exactly at the band gap center [27]. We only investigated this case. Changing the $\varphi$ value, one can effectively tune the spectrum $[28,29]$.

\section{Hybrid Tamm Modes}

When the TP and COTS frequencies in the structure shown in Fig. 1 are matched, the electromagnetic fields of localized modes overlap, which leads to the spectral splitting of the peaks. Two transmittance peaks appear in the spectrum. The splitting value depends on the PPAM thickness (Fig. 4). At $N=40$, the fields overlap very weakly and the splitting does not occur.

Figure 5 shows the spatial electric field intensity distribution in the structure for at an incident radiation wavelength of $\lambda=641 \mathrm{~nm}$ (the case $N=10 \mathrm{in} \mathrm{Fig.} \mathrm{4).} \mathrm{The} \mathrm{field}$ is localized with the maxima at the interfaces. Since the light falls from the left, the field is localized stronger on the left boundary of the structure. With increasing distance to the boundaries, the fields exponentially decay. Such a field distribution confirms the excitation of hybrid modes in the structure.

An important advantage of the proposed model is the use of a CLC, which is known for its high sensitivity. Let us consider the case $d=330 \mathrm{~nm}$. At this sublayer 
thickness, the TPs are excited at wavelengths of 639 and $666 \mathrm{~nm}$ for the TE and TM polarizations of the incident light, respectively $(N=20)$. Changing the helix pitch, we can sequentially hybridize the COTS with both TPs (Fig. 6).

The investigated model based on the hybrid TP-COTS modes can form a basis of lasers with tunable characteristics. As was shown in [34], the laser generation can be obtained using the TP arising at the interface between a multilayer mirror and a silver film. The investigated sample consisted of $40 \mathrm{AlAs}$ and $\mathrm{Ga}_{0,95} \mathrm{Al}_{0,05} \mathrm{As}$ periods; 15 upper periods were $\mathrm{Ga}_{0.9} \mathrm{In}_{0.07} \mathrm{As}$ with quantum dots serving as an active medium. The pumping was performed through the silver layer. It was shown that, at low powers, the generation only occurs at the exciton frequency, while the growth of the latter leads to the generation at the frequency of a Tamm plasmon exciton polariton. It was mentioned that the hybrid character of these modes opens the way to the implementation of novel microlasers that can be excited electrically, with the upper metal layer serving as an electrode. In the model proposed by us, the aforementioned principle of forming an active medium can be implemented. It is worth noting that a feature of the structure based on the hybrid TP-COTS modes is the presence of two TP lines excited for the TE and TM waves. Thus, selecting the emission band of the quantum walls, one can ensure laser generation at two wavelengths simultaneously. The change of the CLC helix pitch (Fig. 7) will be used to control the emission band splitting and obtain the two- $(450<p$ $>350)$ or three-mode $(350<\mathrm{p}<450)$ operation of the device.

\section{CONCLUSIONS}

We proposed a concept of a tunable hybrid Tamm mode-liquid crystal device consisting of a thin metallic film, a multilayer anisotropic mirror, and a cholesteric. Two types of Tamm modes - Tamm plasmons and the chiral optical Tamm state - are excited in the structure. The TPs are localized at the interface between the metallic film and the anisotropic mirror and the COTS is localized at the interface between the anisotropic mirror and the cholesteric. The TPs are excited at two different frequencies for the orthogonal linear polarizations, while the COTS is excited at only one frequency, regardless of the polarization of the incident light. The coupling between the two types of Tamm modes was demonstrated. The spectral characteristics of the proposed device were analytically and numerically calculated. The advantage of the model is the possibility of changing the spectral position of the Tamm state using external factors and, consequently, the hybridization and mode splitting values. The proposed model has a potential for application in a tunable laser operating in the two- or three-mode regime. 


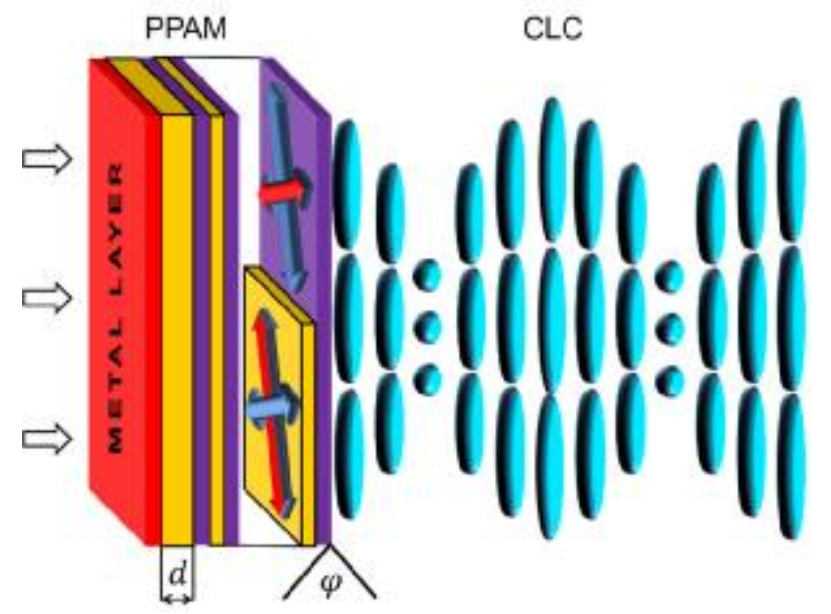

Fig. 1. Schematic of the structure. Color arrows show the direction of optical axes of the PPAM layers.

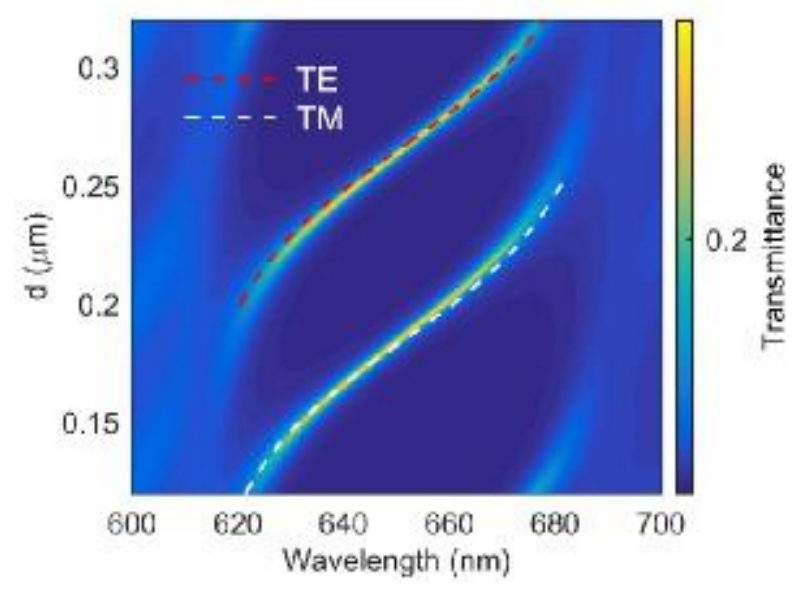

Fig. 2. Transmittance spectrum of the metal-PPAM structure with different thicknesses $d$ for the circularly polarized incident light. White and red lines show positions of the TP peaks for the orthogonal linear polarizations according to Eq. (6). The simulation parameters are $n_{\mathrm{e}}^{m}=1.71, n_{\mathrm{o}}^{m}=$ $1.54,2 a=200 \mathrm{~nm}, N=20, d_{\mathrm{m}}=50 \mathrm{~nm}, \varepsilon_{0}=5, \hbar \omega_{\mathrm{p}}=9 \mathrm{eV}$, and $\hbar \gamma=0.02 \mathrm{eV}$.

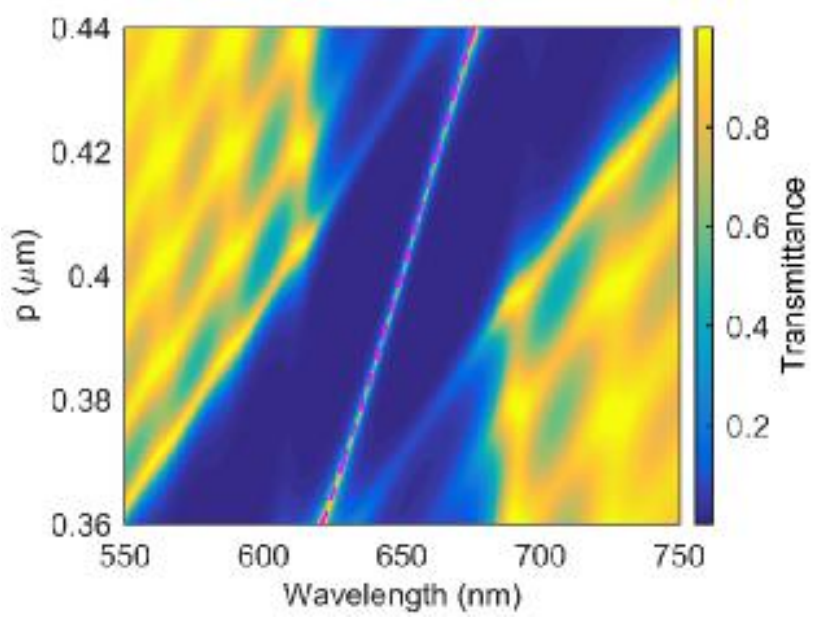


Fig. 3. Transmittance spectrum of the PPAM-CLC structure for the right-hand circularly polarized light at different CLC helix pitches $p$. The magenta line corresponds to the COTS frequency calculated using Eq. (10). The CLC refractive indices are $n_{\mathrm{e}}=1.71$ and $n_{\mathrm{o}}=1.54$, the thickness is $4 \mu$, and the parameters of the metal are the same as in Fig. 2.

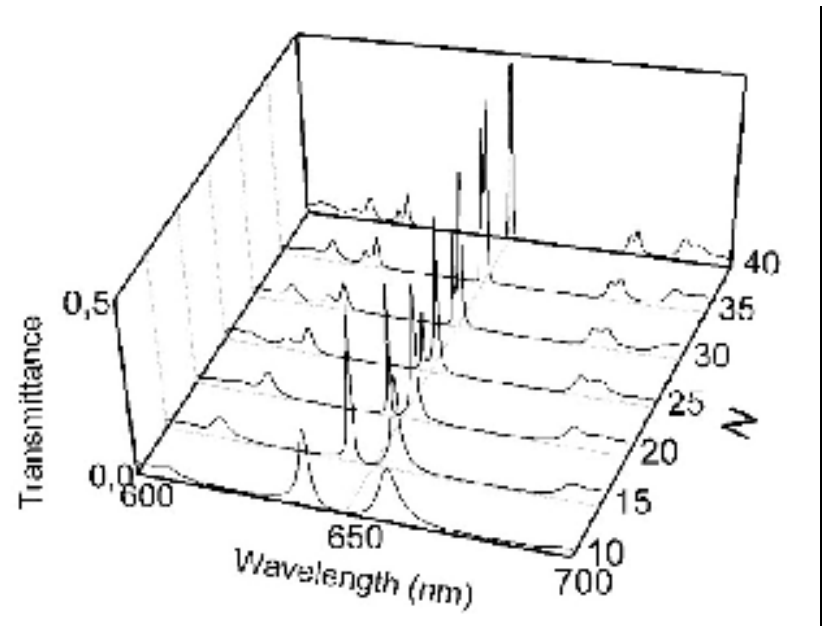

Fig. 4. Dependence of the transmittance spectrum (Fig. 1) on the number of PPAM periods $N$ for the right-hand circular polarization of the incident light. $p=400 \mathrm{~nm}, d=184 \mathrm{~nm}$, and the rest parameters are the same as in Figs. 2 and 3.

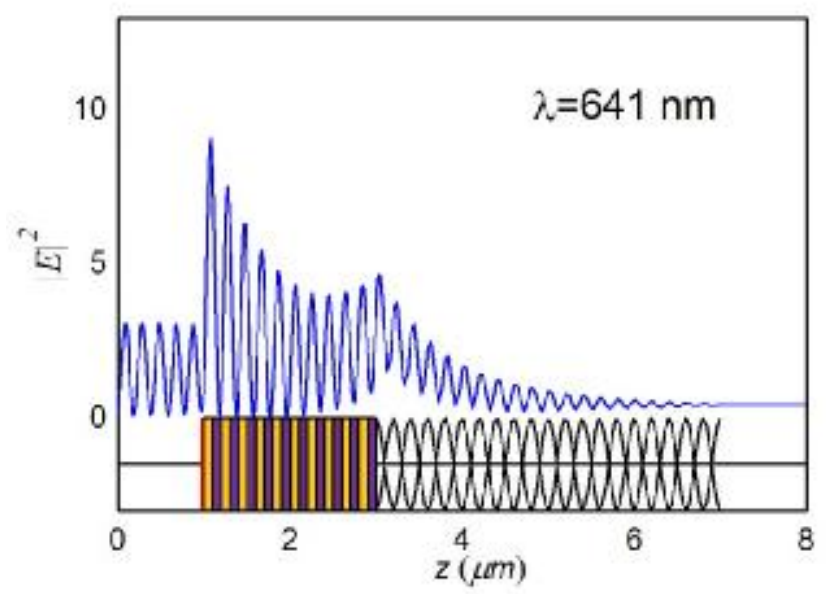

Fig. 5. Local spatial distribution of the squared electric field strength in the structure for the right-hand circular polarization of the incident light. $p=400 \mathrm{~nm}, d=184 \mathrm{~nm}$, and the rest parameters are the same as in Figs. 2 and 3. 


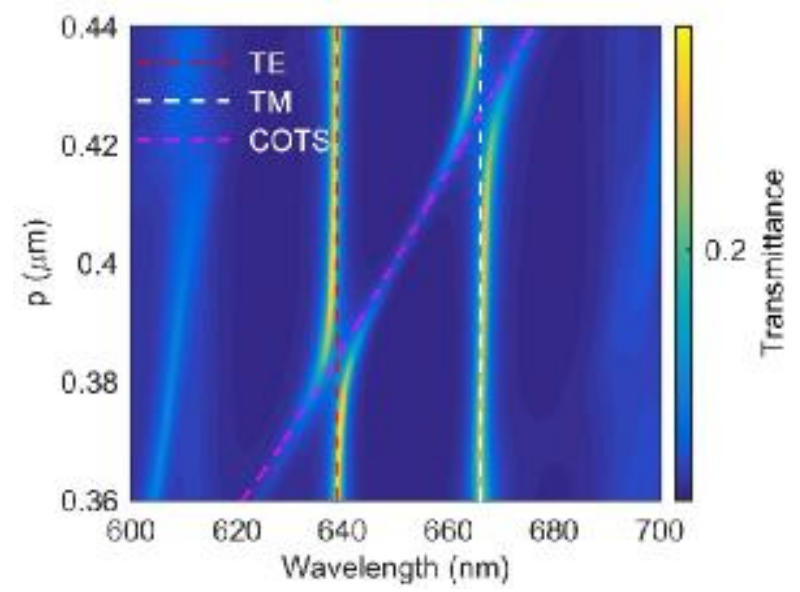

Fig. 6. Transmittance of the structure (Fig. 1) vs CLC helix pitch. The circularly polarized diffracting light falls onto the structure. White and red lines show the positions of single TPs calculated using Eq. (6) for the orthogonal linear polarizations. The magenta line shows the COTS position according to Eq. (10). $p=400 \mathrm{~nm}, d=330 \mathrm{~nm}$, and the rest parameters are the same as in Figs. 2 and 3. 
1. T. Goto, A. V. Dorofeenko, A. M. Merzlikin, A. V. Baryshev, A. P. Vinogradov, M. Inoue, A. A. Lisyansky, and A. B. Granovsky, Phys. Rev. Lett. 101,113902 (2008).

2. M. Kaliteevski, I. Iorsh, S. Brand, R. A. Abram, J. M. Chamberlain, A. V. Kavokin, and I. A. Shelykh, "Tamm plasmon-polaritons: possible electro-magnetic states at the interface of a metal and a dielectric Bragg mirror,” Phys. Rev. B 76, 165415 (2007).

3. A. P. Vinogradov, A. V. Dorofeenko, A. M. Merzlikin, and A. A. Lisyansky, "Surface states in photonic crystals," Phys. Usp. 53, 243-256 (2010).

4. C. Symonds, G. Lheureux, J. P. Hugonin, J. J. Greffet, J. Laverdant, G. Brucoli, A. Lemaitre, P. Senellart, and J. Bellessa, Confined Tamm plasmon lasers, Nano Lett.13, 3179 (2013).

5. Jiménez-Solano A., Galisteo-López J. F., Míguez H. Flexible and Adaptable Light-Emitting Coatings for Arbitrary Metal Surfaces based on Optical Tamm Mode Coupling //Advanced Optical Materials. - 2018. - T. 6. - №. 1. - C. 1700560.

6. Narrowband Wavelength Selective Thermal Emitters by Confined Tamm Plasmon Polaritons ACS Photonics, 2017, 4 (9), pp 2212-2219

7. Multi-channel perfect absorber based on a one-dimensional topological photonic crystal heterostructure with graphene Optics Letters Vol. 43, Issue 17, pp. 4256-4259 (2018) Xi Wang, Yanzhao Liang, Leiming Wu, Jun Guo, Xiaoyu Dai, and Yuanjiang Xiang

8. Bikbaev R. G., Vetrov S. Y., Timofeev I. V. Epsilon-near-zero absorber by tamm plasmon polariton //Photonics. - Multidisciplinary Digital Publishing Institute, 2019. - T. 6. - №. 1. - C. 28.

9. S.-G. Huang, K.-Ping Chen, and S.-C. Jeng, "Phase sensitive sensor on Tamm plasmon devices," Opt. Mater. Exp. 7, 1267-1273 (2017).

10. X.-L. Zhang, J.-F. Song, X.-B. Li, J. Feng, and H.-B. Sun, “Optical Tamm states enhanced broadband absorption of organic solar cells", Appl. Phys. Lett.101, 243901 (2012).

11. Topological insulator based Tamm plasmon polaritons APL Photonics 4, 040801 (2019) Hua Lu1, Yangwu Li, Zengji Yue, Dong Mao, and Jianlin Zhao

12. Zak phase and topological plasmonic Tamm states in one-dimensional plasmonic crystals Lei Wang, Wei Cai, Mengli Bie, Xinzheng Zhang, and Jingjun Xu Optics Express Vol. 26, Issue 22, pp. 28963-28975 (2018)

13. Shie-Chang Jeng Applications of Tamm plasmon-liquid crystal devices LIQUID CRYSTALS 2020

14. Liquid-Crystal Active Tamm-Plasmon Devices Hao-Chi Cheng, Ching-Yung Kuo, Yu-Ju Hung, Kuo-Ping Chen, and Shie-Chang Jeng Phys. Rev. Applied 9, 064034

15. Tamm plasmon photonic crystals: Frombandgap engineering to defect cavity APL Photonics 4, 106101 (2019); Lydie Ferrier, Hai Son Nguyen, Cécile Jamois, Lotfi Berguiga, Clémentine Symonds, JoëlBellessa, and Taha Benyattou

16. Model for confined Tamm plasmon devices Journal of the Optical Society of America B Vol. 36, Issue 1, pp. 125-130 (2019) Mike Adams, Ben Cemlyn, lan Henning, Matthew Parker, Edmund Harbord, and Ruth Oulton 
17. M. Kaliteevski, S. Brand, R. A. Abram, I. Iorsh, A. V. Kavokin, and I. A. Shelykh, "Hybrid states of Tamm plasmons and exciton polaritons,” Appl. Phys. Lett. 95, 251108 (2009).

18. Rahman Sk. S.-U., T. Klein, S. Klembt, J. Gutowski, D. Hommel, anf K.Sebald, "Observation of a hybrid state of Tamm plasmons and microcavity exciton polaritons," Sci. Rep. 6,34392 (2016).

19. B. I. Afinogenov, V. O. Bessonov, A. A. Nikulin, and A. A. Fedyanin, "Observation of hybrid state of Tamm and surface plasmon-polaritons in one-dimensional photonic crystals," Appl. Phys. Lett. 103, 061112 (2013).

20. Pankin, P. S., Vetrov, S. Y., \& Timofeev, I. V. Tunable hybrid Tamm-microcavity states. JOSA B, 34(12), 2633-2639 (2017).

21. Tunable hybrid optical modes in a bounded cholesteric liquid crystal with a twist defect MV Pyatnov, SY Vetrov, IV Timofeev Physical Review E 97 (3), 0327032018

22. Kumar, P. S. Maji, and R. Das, Tamm-plasmon resonance based temperature sensor in a Ta2O5/SiO2 based distributed Bragg reflector, Sens. Actuators A 260, 10 (2017).

23. M. Mehdi Keshavarz and Abbas Alighanbari Terahertz refractive index sensor based on Tamm plasmon-polaritons with grapheme Applied Optics Vol. 58, Issue 13, pp. 3604-3612 (2019)

24. Oleksandr Buchnev, Alexandr Belosludtsev, Victor Reshetnyak, Dean R. Evans and Vassili A. Fedotov Observing and controlling a Tamm plasmon at the interface with a metasurface Nanophotonics 2020; aop

25. Belyakov V. Diffraction Optics of Complex-Structured Periodic Media, Second Edition. Springer, 2019. - P. 300.

26. S. Ya. Vetrov, M. V. Pyatnov, and I. V. Timofeev, "Spectral and polarization properties of a ‘cholesteric liquid crystal—phase plate—metal'structure,” J. Opt. 18, 015103 (2015).

27. Vetrov S. Y., Timofeev I. V., Shabanov V. F. Localized modes in chiral photonic structures //Physics-Uspekhi. - 2020. - T. 63. - №. 1. - C. 33.

28. I. V. Timofeev, P. S. Pankin, S. Y. Vetrov, V. G. Arkhipkin, W. Lee, and V. Y. Zyryanov, "Chiral Optical Tamm States: Temporal Coupled-Mode Theory," Crystals 7, 113 (2017).

29. Chiral Optical Tamm States at the Interface between an All-Dielectric Polarization-Preserving Anisotropic Mirror and a Cholesteric Liquid Crystal NV Rudakova, IV Timofeev, RG Bikbaev, MV Pyatnov, SY Vetrov, W Lee Crystals 9 (10), 5022019

30. Coupled chiral optical Tamm states in cholesteric liquid crystals MV Pyatnov, IV Timofeev, SY Vetrov, NV Rudakova Photonics 5 (4), 302018

31. Yariv A., Yeh P. Optical waves in crystals. - New York : Wiley, 1984. - T. 5.

32. M. Born and E. Wolf, Principles of Optics: Electromagnetic Theory of Propagation, Interference and Diffraction of Light (Pergamon, 1986), Chap. 7.6, p. 325.

33. D. W. Berreman, "Optics in Stratified and Anisotropic Media: 4 X 4-Matrix Formulation”, J. Opt. Soc. Am. 62, 502-510 (1972).

34. C. Symonds, A. Lemaitre, P. Senellart, M. H. Jomaa, S. A. Guebrou, E. Homeyer, G. Brucoli, and J. Bellessa, "Lasing in a hybrid GaAs/silver Tamm structure," Appl. Phys. Lett. 100, 121122 (2012). 